\title{
Clinical-dermoscopic similarities between atypical nevi and early stage melanoma
}

\author{
CRISTINA-RALUCA JITIAN (MIHULECEA) ${ }^{1,2}$, SIMONA FRĂȚILĂ $\breve{~}^{3,4}$ and MARIA ROTARU ${ }^{1,2,5}$
}

\author{
${ }^{1}$ Dermatology Clinic, Emergency Clinical County Hospital of Sibiu, 550245 Sibiu; ${ }^{2}$ Doctoral Studies, \\ 'Victor Babeș' University of Medicine and Pharmacy of Timișoara, 300041 Timișoara; ${ }^{3}$ Faculty of Medicine and Pharmacy, \\ University of Oradea, 410073 Oradea; ${ }^{4}$ Dermatology Department, Emergency Clinical County Hospital of Oradea, \\ 410169 Oradea; ${ }^{5}$ Dermatology Department, Faculty of Medicine, 'Lucian Blaga' University of Sibiu, 550169 Sibiu, Romania
}

Received April 5, 2021; Accepted May 5, 2021

DOI: $10.3892 / \mathrm{etm} .2021 .10286$

\begin{abstract}
Atypical (Clark) nevi are benign tumors that may be considered precursors of melanoma. Many studies acknowledge a linear progression from typical to atypical nevi that eventually transform into melanoma. It is often challenging to differentiate a Clark nevus from melanoma, especially in its early stages, due to their clinical, dermoscopic, and histological resemblance. Dermoscopy is a powerful tool in early melanoma diagnosis, but it is a subjective method of examination. Therefore, the use of dermoscopic algorithms and checklists can overcome this issue. In the case of a difficult diagnosis, since both dermoscopy and histopathological exam are subjective methods of examination, modern molecular biology techniques can be used to distinguish between benign and malignant tumors. This study aimed to test the accuracy of specific clinical and dermoscopic criteria in order to distinguish between benign and malignant tumors, with a secondary objective to provide an overview of the clinical and dermoscopic features of atypical nevi and melanoma. In the present study, dermoscopic algorithms did not necessarily help distinguish benign and malignant tumors but demonstrated that nevi and melanoma have similar characteristics.
\end{abstract}

\section{Introduction}

Melanoma is one of the most aggressive skin cancers that annually claims over 20,000 lives in Europe. The eastern half of European countries report low incidence rates but have a high case fatality, increasing mortality, mostly due to a late diagnosis (1). Several studies report a possible linear progression

Correspondence to: Dr Cristina-Raluca Jitian (Mihulecea), Dermatology Clinic, Emergency Clinical County Hospital of Sibiu, 2-4 Corneliu Coposu Boulevard, 550245 Sibiu, Romania

E-mail: cristina.jitian@yahoo.com

Key words: atypical nevi, melanoma, dermoscopy, histology, early diagnosis from common to atypical nevi that eventually progress to melanoma (2). Moreover, there are two types of melanoma: De novo (DNM) and nevus-associated melanoma $(3,4)$.

Dermoscopy is a non-invasive method of examination that can be used for early melanoma diagnosis and can help differentiate between benign and malignant tumors (5). Still, it is recommended to be used as an adjuvant tool for clinical skin examination $(5,6)$. The 'Chaos and Clues' algorithm is practical and easy to use (7); to date, there are few reports that use this algorithm to distinguish atypical nevi from melanoma.

Atypical nevi are considered cutaneous lesions that identify individuals who are at increased risk of developing melanoma (8). They can have certain dermoscopic features regarding pattern, colors, and clues; their pattern can be typical (reticular/reticular with dots or clods), occasional (structureless hyperpigmented areas in the center and reticular at the periphery), or a combination of reticular lines with/without clods with a structureless skin-colored area (9). The standard colors of Clark nevi are a uniform light-brown or various shades of brown with hyperpigmentation (9). Polychromy can occur with multiple shades of brown or eccentric hyperpigmentation. The specific clues of atypical nevi that can help differentiate them from an early stage melanoma are the presence of reticular lines, usually thin, regular dots and clods, that can appear peripherally in an early phase of growth. Usually, in atypical nevi, the vessels are monomorphic compared to melanoma, where the vessels are irregular and polymorphous (9). In comparison to nevi, melanoma can have a chaotic dermoscopic appearance, but according to several studies, a melanoma in its early stages can be challenging to differentiate from a Clark nevus (10).

Several algorithms can aid in the dermoscopic differentiation between an atypical nevus and an early melanoma, such as the 'ABCD rule', the Menzies method, the 7-point checklist, the 3-point checklist, 'Chaos and clues', and CASH (color, architecture, symmetry, homogeneity) $(11,12)$. Each algorithm is unique, with a different sensitivity and specificity in the diagnosis of melanocytic lesions. A study conducted by Carrera et al demonstrated that the Menzies method was the most sensitive for melanoma diagnosis (95.1\%) but had the lowest specificity (24.8\%), while the ABCD rule algorithm had the highest specificity (59.4\%) (11). 
This study aimed to diagnose and differentiate atypical nevi from early melanomas using specific clinical and dermoscopic criteria, including the 'Chaos and clues' algorithm introduced by Rosendahl et al (7).

\section{Materials and methods}

We present an observational, retrospective study of 103 melanocytic lesions dermatologically monitored between 2017 and 2019 at the Clinical Hospital and private Dermatology offices of Sibiu and Oradea County. The lesions were examined clinically, dermoscopically, and histopathologically. The data collected were related to the assessed clinical and dermoscopic features of the lesions, which were examined and revised by three evaluators. The dimensions of the tumors were measured in millimeters $(\mathrm{mm})$, and the dermoscopic images were evaluated for the presence/absence of chaos and any of Rosendahl's et al (7) clues for malignancy. The colors of the lesions and clinical criteria were also recorded. As this was an observational study, it was exempted from the Ethics Committee of Sibiu's County Clinical Hospital review (Sibiu, Romania).

Statistical analysis. Data were collected and tabulated on Microsoft Excel spreadsheets for statistical analysis [calculation of the prevalence of the variables (\%), the median size of the lesions, and the number of colors]. The variables are expressed in numbers and percentages to simplify the statistical process.

\section{Results}

The selected tumors were examined clinically, through dermoscopy, and a part of them were confirmed histopathologically. Out of 103 lesions, only $45.63 \%$ (47 lesions) were excised and had a histopathological exam, partly because most patients refused to have an interventional treatment and preferred to be followed-up at 3-6 months. Among the excised lesions, $70.21 \%$ (33 lesions) were atypical nevi, $14.89 \%$ (7 lesions) melanomas, and $14.89 \%$ (7 lesions) common nevi. Regarding the pathologically confirmed melanomas, the clinical and dermoscopic examinations were in accordance with the pathology reports. The majority of the melanoma subtypes was lentigo maligna melanoma (LMM) with a median Breslow index (BI) of $1.28 \mathrm{~mm}$, followed by superficial spreading melanoma (SSM) with an IB of 0.5-1 mm. Two achromic melanomas (ungual and SSM) were also observed. A percentage of $48.54 \%$ of the selected lesions belonged to patients with atypical mole syndrome (AMS). Clinically, the 'ABCD rule' (A-asymmetry, B-border, C-color, D-diameter) was used to assess the most frequent criteria found in the histologically confirmed benign and malignant tumors. We obtained the following: The ' $\mathrm{E}$ ' $(100.00 \%)$ and 'B' $(81.81 \%)$ were the most frequently encountered criteria in the population with atypical nevi (Fig. 1). All the ABCDE criteria were present in the melanoma tumors $(100.00 \%)$; the criteria 'A', 'B', and ' $\mathrm{C}$ ' were the most frequently encountered in the biopsied common nevi, with a percentage of $85.71 \%$, which clinically justified the decision to biopsy them.

The tumors were also assessed using the 'Chaos and clues' algorithm and specific dermoscopy criteria to differentiate
Table I. Size and colors of atypical nevi and melanoma.

\begin{tabular}{|c|c|}
\hline Atypical nevi features $\%$ & Melanoma features $\%$ \\
\hline Colors & Colors \\
\hline Blue $3.03 \%$ & Blue $57.14 \%$ \\
\hline Light brown $100.00 \%$ & Light brown $85.71 \%$ \\
\hline Dark brown $96.96 \%$ & Dark brown $85.71 \%$ \\
\hline Black $57.57 \%$ & Black $71.42 \%$ \\
\hline White $12.12 \%$ & White $71.42 \%$ \\
\hline Grey $24.24 \%$ & Grey $71.42 \%$ \\
\hline Red $24.24 \%$ & Red $42.85 \%$ \\
\hline Purple $0.00 \%$ & Purple $42.85 \%$ \\
\hline Yellow $9.09 \%$ & Yellow $28.57 \%$ \\
\hline Orange $0.00 \%$ & Orange $14.28 \%$ \\
\hline Median no. of colors 3.27 & Median no. of colors 5.71 \\
\hline Size & Size \\
\hline$<5 \mathrm{~mm}=39.39 \%$ & $<5 \mathrm{~mm}=0.00 \%$ \\
\hline $5-10 \mathrm{~mm}=42.42 \%$ & $5-10 \mathrm{~mm}=14.28 \%$ \\
\hline$>10 \mathrm{~mm}=18.18 \%$ & $>10 \mathrm{~mm}=85.71 \%$ \\
\hline Median size of $6.46 \mathrm{~mm}$ & Median size of $16.42 \mathrm{~mm}$ \\
\hline Total no. of lesions $=33$ & Total no. of lesions $=7$ \\
\hline
\end{tabular}

no., number; mm, millimeters.

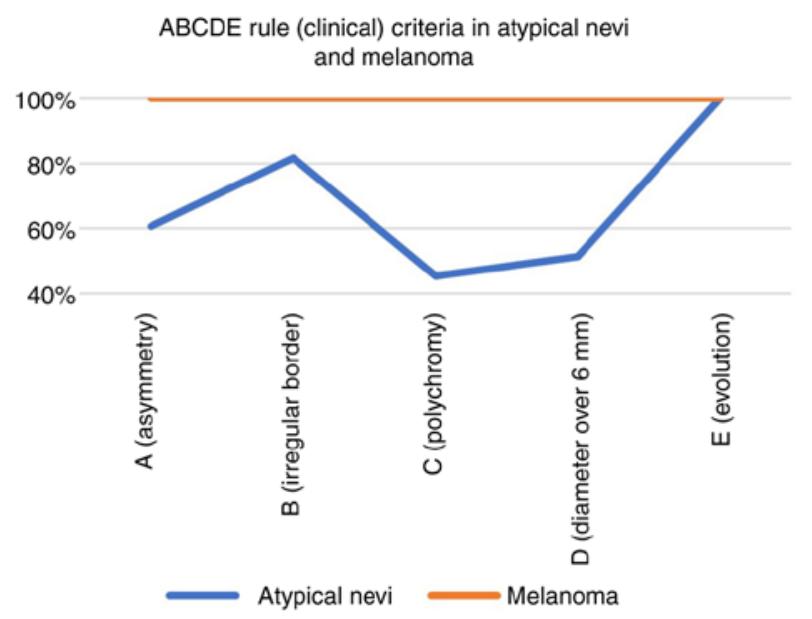

Figure 1. 'ABCDE rule' criteria in atypical nevi and melanoma.

benign from malignant lesions and observe the most specific clues for atypical nevi and melanoma.

First, the lesions were assessed by the 'chaos' (asymmetry of pattern or color) criterion, and out of 103 examined tumors, a percentage of $42.71 \%$ had a chaotic appearance and were analyzed further to see which clues of malignancy were the most detected. A percentage of $66.66 \%$ of the atypical nevi had a chaotic appearance, with a median of 2.21 out of 9 clues. The most encountered clues were polymorphous vessels $(63.63 \%)$ and reticular/branched thick lines (39.39\%), while less frequent were the radial lines/pseudopods, $9.09 \%$ (Fig. 2). Parallel lines, ridges (acral), or chaotic lines (nails) were not present in the atypical nevi population. 


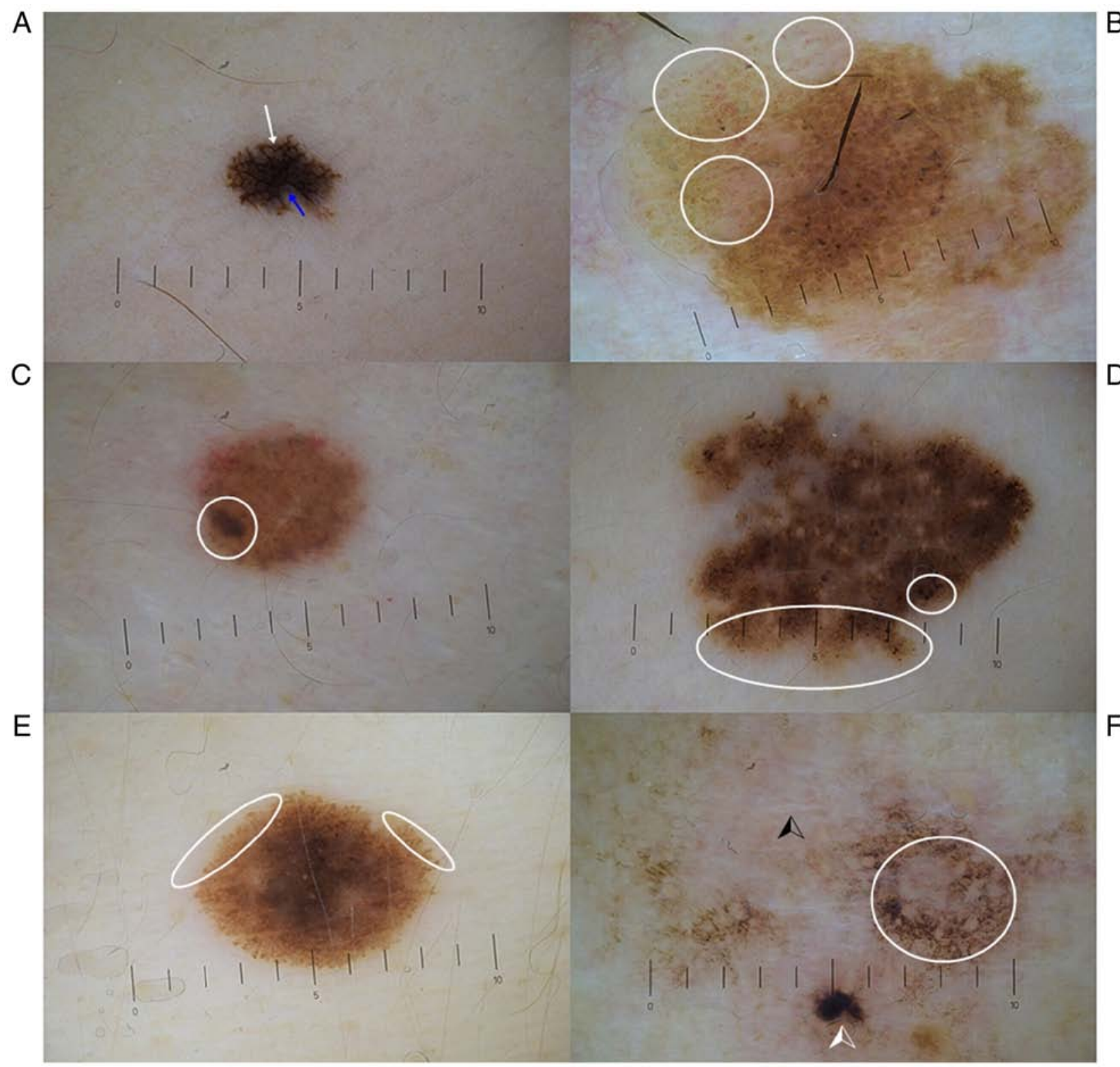

Figure 2. 'Chaos and clues' in atypical nevi and melanoma. (A) Atypical nevus with thick lines (white arrow) and grey-blue structures (blue arrow). (B) Atypical nevus with polymorphous vessels (white circles). (C) Atypical nevus with eccentrical structure (white circle). (D) Atypical nevus with peripheral black dots. (E) Atypical nevus with pseudopods. (F) Melanoma has chaos, eccentrical black structure (white arrowhead), white lines (black arrowhead), and grey structures (white circle).

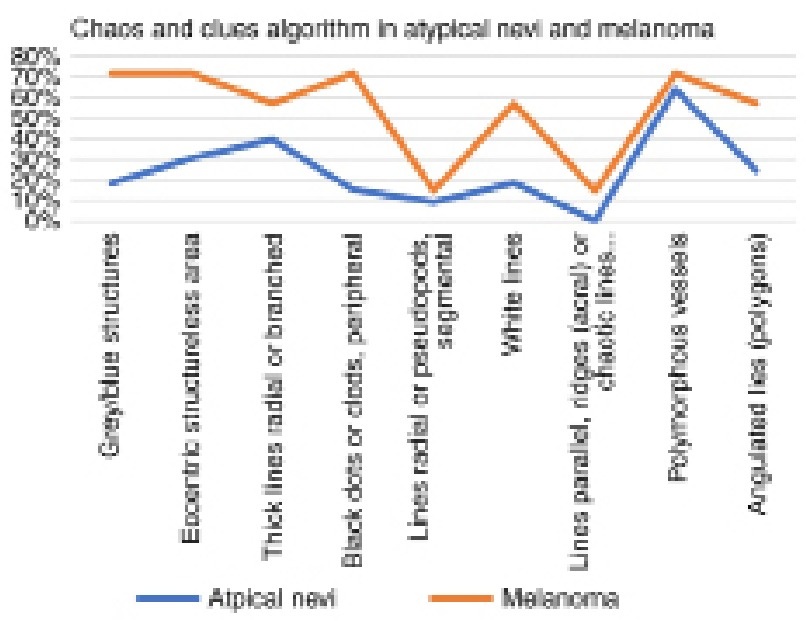

Figure 3. 'Chaos and clues' algorithm in atypical nevi and melanoma.
All melanoma tumors presented chaos, with a median of 4.85 out of 9 clues.

The most specific clues for melanoma were polymorphous vessels, grey/blue structures, eccentric structureless area, peripheral black dots/clods $(71.42 \%)$, followed by white lines, angulated lines, thick reticular/branched lines (57.14\%) (Fig. 3).

All atypical nevi had more than one color (median of 3.27 colors), and the most prevalent colors were: Light brown (100.00\%), dark brown (96.96\%), and black (57.57\%). The studied dysplastic nevi had a median size of $6.46 \mathrm{~mm}$. All melanomas had more than one color with a median of 5.71 colors; the most encountered colors were light and dark brown $(85.71 \%)$, followed by black, white, and grey $(71.42 \%)$. The median size of the melanoma tumors was $16.42 \mathrm{~mm}$, most of them having a size $>10 \mathrm{~mm}(85.71 \%)$ (Table I). 


\section{Discussion}

Dermoscopy is a helpful method of examination that can improve the early diagnosis of melanoma compared to clinical examination. The 'Chaos and clues' algorithm was created to be applied to melanocytic lesions, and it is used to detect malignancy. According to Rosendahl et al, this algorithm has a sensitivity of $90.60 \%$ and a specificity of $62.70 \%$ for malignancy diagnosis (7). However, the lesions should first be examined through dermoscopy for chaos (asymmetry of pattern or color).

When chaos is encountered, the clinician should search for at least one clue of malignancy: Grey/blue structures, eccentric structureless area, thick reticular/branched lines, peripheral black dots/clods, segmental pseudopods/radial lines, white lines, parallel lines, ridges (acral) or chaotic lines (nails), polymorphous vessels, angulated lines (polygons). If at least one clue of malignancy is present, the lesion is indicated for excision. All malignant tumors examined by dermoscopy presented 'chaos', similar to Ramji et al (13).

The most specific melanoma criteria of the present study included polymorphous vessels, grey/blue structures, eccentric structureless areas, peripheric black dots/clods (71.42\%), followed by white lines, thick reticular/branched lines, and angulated lines (57.14\%). In atypical nevi, white lines were uncommon, similar to a study by Verzi et al, which reported that white streaks are highly unusual and more specific to melanomas; 31 out of 144 melanomas presented white lines (22\%) (14).

According to a study by Marghoob et al, white lines (structures) have a specificity of $80.6 \%$ for melanoma (2.5 to 9.7 OR) (15). Recently, the angulated lines clue was added to the original 'Chaos and clues' algorithm by Jaimes and coworkers, as they consider that it is a specific feature of flat melanomas on chronic-sun damaged skin (16). In the present study, angulated lines were present in $57.14 \%$ of the melanoma population and $24.24 \%$ of the atypical nevi, which demonstrates their specificity for malignant tumors. Carrera et al report that structureless areas were detected in $47.6 \%$ of the nevi examined, while in our study, we observed this criterion (eccentric structureless areas) in $30.30 \%$ of the atypical nevi, in $50.00 \%$ of the common nevi, and $71.42 \%$ of the melanoma tumors (11). A study by Lallas et al suggested that irregular hyperpigmented areas represent melanoma indicators, compared to atypical nevi (17); the same was demonstrated in our study regarding the eccentrical structureless areas.

According to Rezze et al, the 'ABCDE rule' can be useful in the clinical diagnosis of atypical nevi (18). Clinically, our study showed that all the biopsied atypical nevi had the 'E' (evolution) criterion of the 'ABCDE rule', which outlines the importance of periodic clinical and dermoscopic examination of these lesions since many patients can be unaware of changes in their nevi. In a study by Rivers et al, 13 out of 16 patients were unaware of any change in their cutaneous lesions (8).

The color and size analyses showed that melanomas present more colors (median of 3.27 colors in NA vs. 5.71 in $\mathrm{MM}$ ) and are more prominent (median of $6.46 \mathrm{~mm}$ in NA vs. $16.42 \mathrm{~mm}$ in MM) than atypical nevi. All melanomas had more than one color, and the most encountered colors were light and dark brown (85.71\%), followed by white, black, and grey $(71.42 \%)$, similar to the study by Ramji et al in which the most frequent colors were light and dark brown (100 and 98\%) and grey (75\%) (13).

Several risk factors can drive a common nevus to malignancy since there seems to be a linear progression from atypical nevi to melanoma (2). The most important risk factors are UV radiation and genetics. According to a study by Fechete $\mathrm{et} \mathrm{al}$, melanoma patients are more likely to have fair skin, freckles, a large number of nevi (over 20), atypical nevi, frequently or partially outdoor occupations, had over three sunburns during early life, or had used sun beds (19). In our study, $48.54 \%$ of the patients had multiple nevi (atypical mole syndrome).

According to different studies, most melanomas develop de novo (DNM), while nevus-associated melanomas (NAM) are less frequent $(3,4)$. Vezzoni et al report two types of NAM: Melanoma that develops in the center of a mole (probably a congenital nevus) and melanoma that arises next to the mole (dysplastic nevus) (20). In our study, three melanomas had eccentrical black pigmentation (possible melanomas developed from atypical nevi), and only one had central pigmentation (possible melanoma developed from a congenital nevus). According to Haenssle et al, patients with multiple nevi and without previous melanomas or atypical mole syndrome had a higher frequency of NAM (21). Alendar and Kittler (22) and Lin et al (23) report that survival does not differ significantly between patients with NAM and patients with DNM). On the contrary, Cymerman et al suggest that patients with DNM have a more unsatisfactory outcome than NAM patients (24).

Our study's limitations are the relatively low number of the examined tumors and the subjectivity of the clinical and dermoscopic assessment of the melanocytic lesions, which is why some results may differ from other studies. The 'Chaos and clues' algorithm did not necessarily help distinguish between benign and malignant tumors but helped demonstrate that nevi and melanoma have similar characteristics. Possibly, confocal laser scanning microscopy (CLSM) would have aided in establishing a prompt diagnosis, as it is known to differentiate between different skin tumors (25), but unfortunately, we do not have such equipment in our daily practice.

In conclusion, all suspicious pigmented lesions with a recent change in history should be monitored carefully through clinical and dermoscopic examination. Most atypical nevi can mimic an early melanoma; that is why a highly atypical pigmented lesion should be excised after a thorough clinical and dermoscopic examination. Early detection of melanoma is critical in individuals with atypical nevi because they have an increased risk of developing melanoma compared to the general population (8). The presence of chaos/malignancy clues should lead to the excision of the assessed lesion to exclude malignancy (13). Certain authors report a linear progression of common nevi to atypical nevi that may transform into melanoma in time (2). A malignant transformation of a nevus cannot be predicted, but periodical dermoscopic follow-up can help differentiate a nevus from an early melanoma (26). It is often difficult to differentiate between atypical nevi and early melanoma through the usual examination methods (dermoscopy, histology). In this case, modern techniques of molecular biology or CLSM can be used to distinguish benign from malignant tumors (melanoma). Moreover, investigating the molecular biology of melanoma or using the CLSM could 
help in finding the best therapeutic approach and to possibly identify new therapies $(25,27)$.

\section{Acknowledgements}

Not applicable.

\section{Funding}

No funding was received.

\section{Availability of data and materials}

The datasets used or analyzed during the present study are available from the corresponding author on reasonable request revision of the manuscript for important intellectual content.

\section{Authors' contributions}

CRJM was responsible for the manuscript design, conception, drafting, analysis, and interpretation of the data. SF contributed to the manuscript drafting, data acquisition, and critical revision of the manuscript. MR contributed to the manuscript's conception and design, analysis, acquisition, and interpretation of the data, manuscript drafting, and critical revision of the manuscript for important intellectual content. All authors contributed significantly to this publication and read and approved the final manuscript.

\section{Ethics approval and consent to participate}

As this was an observational study, it was exempted from the Ethics Committee of Sibiu's Emergency Clinical County Hospital review (Sibiu, Romania).

\section{Patient consent for publication}

Not applicable.

\section{Competing interests}

The authors declare that they have no competing interests.

\section{References}

1. Forsea AM: Melanoma epidemiology and early detection in europe: Diversity and disparities. Dermatol Pract Concept 10: e2020033, 2020.

2. Shain AH and Bastian BC: From melanocytes to melanomas. Nat Rev Cancer 16: 345-358, 2016.

3. Pampena R, Kyrgidis A, Lallas A, Moscarella E, Argenziano G and Longo C: A meta-analysis of nevus-associated melanoma: Prevalence and practical implications. J Am Acad Dermatol 77: 938-945.e4, 2017.

4. Pandeya N, Kvaskoff M, Olsen CM, Green AC, Perry S, Baxter C, Davis MB, Mortimore R, Westacott L, Wood D, et al: Factors related to nevus-associated cutaneous melanoma: A case-case study. J Invest Dermatol 138: 1816-1824, 2018.

5. Gniadecki R and Mourad A: Differentiating malignant melanoma from other lesions using dermoscopy. Can Fam Physician 65 412-414, 2019

6. Rotaru M, Jitian CR and Iancu GM: A 10-year retrospective study of melanoma stage at diagnosis in the academic emergency hospital of Sibiu county. Oncol Lett 17: 4145-4148, 2019.
7. Rosendahl C, Cameron A, McColl I and Wilkinson D: Dermatoscopy in routine practice-'chaos and clues'. Aust Fam Physician 41: 482-487, 2012.

8. Rivers JK, Kopf AW, Vinokur AF, Rigel DS, Friedman RJ, Heilman ER and Levenstein M: Clinical characteristics of malignant melanomas developing in persons with dysplastic nevi. Cancer 65: 1232-1236, 1990.

9. Kittler H, Rosendahl C, Cameron A and Tschandl P: Dermatoscopy pattern analysis of pigmented and non-pigmented lesions. 2nd edition. Facultas Verlags, 2016.

10. Rose SE, Argenziano G and Marghoob AA: Melanomas difficult to diagnose via dermoscopy. G Ital Dermatol Venereol 145: 111-126, 2010.

11. Carrera C, Marchetti MA, Dusza SW, Argenziano G, Braun RP, Halpern AC, Jaimes N, Kittler HJ, Malvehy J, Menzies SW, et al: Validity and reliability of dermoscopic criteria used to differentiate nevi from melanoma: A web-based international dermoscopy society study. JAMA Dermatol 152: 798-806, 2016.

12. Weber P, Tschandl P, Sinz C and Kittler H: Dermatoscopy of neoplastic skin lesions: Recent advances, updates and revisions. Curr Treat Options Oncol 19: 56, 2018.

13. Ramji R, Valdes-Gonzalez G, Oakley A and Rademaker M: Dermoscopic 'Chaos and Clues' in the diagnosis of melanoma in situ. Australas J Dermatol 59: 201-205, 2018.

14. Verzi AE, Quan VL, Walton KE, Martini MC, Marghoob AA, Garfield EM, Kong BY, Isales MC, VandenBoom T, Zhang B, et al: The diagnostic value and histologic correlate of distinct patterns of shiny white streaks for the diagnosis of melanoma: A retrospective, case-control study. J Am Acad Dermatol 78: 913-919, 2018.

15. Marghoob NG, Liopyris K and Jaimes N: Dermoscopy: A review of the structures that facilitate melanoma detection. J Am Osteopath Assoc 119: 380-390, 2019.

16. Jaimes N, Marghoob AA, Rabinovitz H, Braun RP, Cameron A, Rosendahl C, Canning G and Keir J: Clinical and dermoscopic characteristics of melanomas on nonfacial chronically sun-damaged skin. J Am Acad Dermatol 72: 1027-1035, 2015.

17. Lallas A, Longo C, Manfredini M, Benati E, Babino G Chinazzo C, Apalla Z, Papageorgiou C, Moscarella E, Kyrgidis A and Argenziano G: Accuracy of dermoscopic criteria for the diagnosis of melanoma in situ. JAMA Dermatol 154: 414-419, 2018.

18. Rezze GG,Leon A and Duprat J: Dysplastic nevus (atypical nevus). An Bras Dermatol 85: 863-871, 2010 (In English, Portuguese).

19. Fechete O, Ungureanu L, Șenilă S, Vornicescu D, Dănescu S, Vasilovici A, Candrea E, Vesa S,C and Cosgarea R: Risk factors for melanoma and skin health behaviour: An analysis on Romanian melanoma patients. Oncol Lett 17: 4139-4144, 2019.

20. Vezzoni R, Conforti C, Vichi S, Giuffrida R, Retrosi C, Magaton-Rizzi G, Di Meo N, Pizzichetta MA and Zalaudek I: Is there more than one road to nevus-associated melanoma? Dermatol Pract Concept 10: e2020028, 2020.

21. Haenssle HA, Mograby N, Ngassa A, Buhl T, Emmert S, Schön MP, Rosenberger A and Bertsch HP: Association of patient risk factors and frequency of nevus-associated cutaneous melanomas. JAMA Dermatol 152: 291-298, 2016.

22. Alendar $\mathrm{T}$ and Kittler $\mathrm{H}$ : Morphologic characteristics of nevi associated with melanoma: A clinical, dermatoscopic and histopathologic analysis. Dermatol Pract Concept 8: 104-108, 2018.

23. Lin WM, Luo S, Muzikansky A, Lobo AZ, Tanabe KK, Sober AJ, Cosimi AB, Tsao H and Duncan LM: Outcome of patients with de novo versus nevus-associated melanoma. J Am Acad Dermatol 72: 54-58, 2015.

24. Cymerman RM, Shao Y, Wang K, Zhang Y, Murzaku EC, Penn LA, Osman I and Polsky D: De novo vs nevus-associated melanomas: Differences in associations with prognostic indicators and survival. J Natl Cancer Inst 108: djw121, 2016.

25. Ilie MA, Caruntu C, Lupu M, Lixandru D, Tampa M, Georgescu SR, Bastian A, Constantin C, Neagu M, Zurac SA and Boda D: Current and future applications of confocal laser scanning microscopy imaging in skin oncology. Oncol Lett 17: 4102-4111, 2019.

26. Rotaru M, Nati AE, Avrămoiu I, Grosu F and Mălăescu GD: Digital dermoscopic follow-up of 1544 melanocytic nevi. Rom J Morphol Embryol 56: 1467-1472, 2015.

27. Surcel M, Căruntu C, Tampa M, Matei C, Pițuru S, Georgescu SR, Constantin C, Zurac $\mathrm{C}$ and Neagu M: Adrenergic modulation of melanoma cells proliferation. Farmacia 66, 820-825, 2018.

This work is licensed under a Creative Commons Attribution-NonCommercial-NoDerivatives 4.0 International (CC BY-NC-ND 4.0) License. 